\title{
Advances in Modal Analysis Using a Robust and Multiscale Method
}

\author{
Cécile Picard, ${ }^{1}$ Christian Frisson, ${ }^{2}$ François Faure, ${ }^{3}$ George Drettakis, ${ }^{1}$ and Paul G. Kry ${ }^{4}$ \\ ${ }^{1}$ REVES/INRIA, BP 93, 06902 Sophia Antipolis, France \\ ${ }^{2}$ TELE Lab, Université catholique de Louvain (UCL), 1348 Louvain-la-Neuve, Belgium \\ ${ }^{3}$ EVASION/INRIA/LJK, Rhône-Alpes Grenoble, France \\ ${ }^{4}$ SOCS, McGill University, Montreal, Canada H3A 2 A7 \\ Correspondence should be addressed to Cécile Picard, cecile.picard@sophia.inria.fr
}

Received 1 March 2010; Revised 29 June 2010; Accepted 18 October 2010

Academic Editor: Xavier Serra

Copyright (C) 2010 Cécile Picard et al. This is an open access article distributed under the Creative Commons Attribution License, which permits unrestricted use, distribution, and reproduction in any medium, provided the original work is properly cited.

\begin{abstract}
This paper presents a new approach to modal synthesis for rendering sounds of virtual objects. We propose a generic method that preserves sound variety across the surface of an object at different scales of resolution and for a variety of complex geometries. The technique performs automatic voxelization of a surface model and automatic tuning of the parameters of hexahedral finite elements, based on the distribution of material in each cell. The voxelization is performed using a sparse regular grid embedding of the object, which permits the construction of plausible lower resolution approximations of the modal model. We can compute the audible impulse response of a variety of objects. Our solution is robust and can handle nonmanifold geometries that include both volumetric and surface parts. We present a system which allows us to manipulate and tune sounding objects in an appropriate way for games, training simulations, and other interactive virtual environments.
\end{abstract}

\section{Introduction}

Our goal is to realistically model sounding objects for animated realtime virtual environments. To achieve this, we propose a robust and flexible modal analysis approach that efficiently extracts modal parameters for plausible sound synthesis while also focusing on efficient memory usage.

Modal synthesis models the sound of an object as a combination of damped sinusoids, each of which oscillates independently of the others. This approach is only accurate for sounds produced by linear phenomena, but can compute these sounds in realtime. It requires the computation of a partial eigenvalue decomposition of the system matrices of the sounding object, which can be expensive for large complex systems. For this reason, modal analysis is performed in a preprocessing step. The eigenvalues and eigenvectors strongly depend on the geometry, material and scale of the sounding object. In general, complex sounding objects, that is, with detailed geometries, require a large set of eigenvalues in order to preserve the sound map, that is, the changes in sound across the surface of the sounding object. This processing step can be subject to robustness problems. This is even more the case for nonmanifold geometries, that is, geometries where one edge is shared by more than two faces. Finally, available approaches manage memory usage in realtime by only pruning part of modal parameters according to the characteristics of the virtual scene (e.g., foreground versus background), without specific consideration regarding the objects' sound modelling. Additional flexibility in the modal analysis itself is thus needed.

We propose a new approach to efficiently extract modal parameters for any given geometry, overcoming many of the aforementioned limitations. Our method employs bounding voxels of a given shape at arbitrary resolution for hexahedral finite elements. The advantages of this technique are the automatic voxelization of a surface model and the automatic tuning of the finite element method (FEM) parameters based on the distribution of material in each cell. A particular advantage of this approach is that we can easily deal with nonmanifold geometry which includes both volumetric and surface parts (see Section 5). These kinds of geometries cannot be processed with traditional approaches which use 
a tetrahedralization of the model (e.g., [1]). Likewise, even with solid watertight geometries, complex details often lead to poorly shaped tetrahedra and numerical instabilities; by contrast, our approach does not suffer from this problem. Our specific contribution is the application of the multiresolution hexahedral embedding technique to modal analysis for sound synthesis. Most importantly, our solution preserves variety in what we call the sound map.

The remainder of this paper is organized as follows. Related work is presented in Section 2. Our method is then explained in Section 3. A validation is presented in Section 4. Robustness and multiscale results are discussed in Section 5, then realtime experimentation is presented in Section 6. We finally conclude in Section 7.

\section{Background}

2.1. Related Work. The traditional approach to creating soundtracks for interactive physically based animations is to directly playback prerecorded samples, for instance, synchronized with the contacts reported from a rigid-body simulation. Due to memory constraints, the number of samples is limited, leading to repetitive audio. Moreover, matching sampled sounds to interactive animation is difficult and often leads to discrepancies between the simulated visuals and their accompanying soundtrack. Finally, this method requires each specific contact interaction to be associated with a corresponding prerecorded sound, resulting in a timeconsuming authoring process.

Work by Adrien [2] describes how effective digital sound synthesis can be used to reconstruct the richness of natural sounds. There has been much work in computer music [3-5] and computer graphics [1, 6, 7] exploring methods for generating sound based on physical simulation. Most approaches target sounds emitted by vibrating solids. Physically based sounds require significantly more computation power than recorded sounds. Thus, bruteforce sound simulation cannot be used for realtime sound synthesis. For interactive simulations, a widely used solution is to apply vibrational parameters obtained through modal analysis. Modal data can be obtained from simulations $[1,7]$ or extracted from recorded sounds of real objects [6]. The technique presented in this paper is more closely related to the work of O'Brien et al. [1], which extends modal analysis to objects that are neither simple shapes nor available to be measured.

The computation time required by current methods to preprocess the modal analysis prevents it from being used for realtime rendering. As an example, the actual cost of computing the partial eigenvalue decomposition using a tetrahedralization in the case of a bowl with 274 vertices and generating 2426 tetrahedra is 5 minutes with a $2.26 \mathrm{GHz}$ Intel Core Duo. Work of Bruyns-Maxwell and Bindel [8] address interactive sound synthesis and how the change of the shape of a finite element model affects the sound emission. They highlight that it is possible to avoid the recomputation of the synthesis parameters only for moderate changes. There has been much work in controlling the computational expense of modal synthesis, allowing the simultaneous handling of a large variety of sounding objects $[9,10]$. However, to be even more efficient, flexibility should be included in the design of the model itself, in order to control the processing. Thus, modal synthesis should be further developed in terms of parametric control properties. Our technique tackles computational efficiency by proposing a multiscale resolution approach of modal analysis, managing the amount of modal data according to memory requirements.

The use of physics engines is becoming much more widespread for animated interactive virtual environments. The study from Menzies [11] address the pertinence of physical audio within physical computer game environment. He develops a library whose technical aspects are based on practical requirements and points out that the interface between physics engines and audio has often been one of the obstacles for the adoption of physically based sound synthesis in simulations. O'Brien et al. [12] employed finite elements simulations for generating both animated videos and audio. However, the method requires large amounts of computation, and cannot be used for realtime manipulation.

2.2. Modal Synthesis. Modal sound synthesis is a physically based approach for modelling the audible vibration modes of an object. As any kind of additive synthesis, it consists of describing a source as the sum of many components [13]. More specifically, the source is viewed as a bank of damped harmonic oscillators which are excited by an external stimulus and the modal model is represented with the vector of the modal frequencies, the vector of the decay rates and the matrix of the gains for each mode at different locations on the surface of the object. The frequencies and dampings of the oscillators are governed by the geometry and material properties of the object, whereas the coupling gains of the modes are determined by the mode shapes and are dependent on the contact location on the object [6].

Modes are computed through an analysis of the governing equations of motion of the sounding system. The natural frequencies are determined assuming the dynamic response of the unloaded structure, with the equation of motion. A system of $n$ degrees of freedom is governed by a set of $n$ coupled ordinary differential equations of second order. In modal analysis, the deformation of the system is assumed to be a linear combination of normal modes, uncoupling the equations of motion. The solution for object vibration can be thus easily computed. To decouple the damped system into single degree-of freedom oscillators, Rayleigh damping is generally assumed (see, for instance, [14]).

The response of a system is usually governed by a relatively small part of the modes, which makes modal superposition a particularly suitable method for computing the vibration response. Thus, if the structural response is characterized by $k$ modes, only $k$ equations need to be solved. Finally, the initial computational expense in calculating the modes and frequencies is largely offset by the savings obtained in the calculation of the response.

Modal synthesis is valid only for linear problems, that is, simulations with small displacements, linear elastic materials, and no contact conditions. If the simulation presents 
nonlinearities, significant changes in the natural frequencies may appear during the analysis. In this case, direct integration of the dynamic equation of equilibrium is needed, which requires much more computational effort. For our approach, the calculations for modal parameters are similar to the ones presented in the paper of O'Brien et al. [1].

\section{Method}

In the case of small elastic deformations, rigid motion of an object does not interact with the objects's vibrations. On the other hand, we assume that small-amplitude elastic deformations will not significantly affect the rigid-body collisions between objects. For these reasons, the rigid-body behavior of the objects can be modeled in the same way as animation without audio generation.

3.1. Deformation Model. In most approaches, the deformation of the sounding object typically need to be simulated. Instead of directly applying classical mechanics to the continuous system, suitable discrete approximations of the object geometry can be performed, making the problem more manageable for mathematical analysis. A variety of methods could be used, including particle systems $[3,7]$ that decompose the structure into small pair-like elements for solving the mechanics equations, or Boundary Element Method (BEM) that computes the equations on the surface (boundary) of the elastic body instead of on its volume (interior), allowing reflections and diffractions to be modeled [15, 16]. The Finite Element Method (FEM) is commonly used to perform modal analysis, which in general gives satisfactory results. Similar to particle systems, FEM discretizes the actual geometry of the structure using a collection of finite elements. Each finite element represents a discrete portion of the physical structure and the finite elements are joined by shared nodes. The collection of nodes and finite elements is called a mesh. The tetrahedral finite element method has been used to apply classical mechanics [1]. However, tetrahedral meshes are computationally expensive for complex geometries, and can be difficult to tune. As an example, in the tetrahedral mesh generator Tetgen (http://tetgen.berlios.de/), the mesh element quality criterion is based on the minimum radius-edge ratio, which limits the ratio between the radius of the circumsphere of the tetrahedron and the shortest edge length. Based on this observation, we choose a finite elements approach whose volume mesh does not exactly fit the object.

We use the method of Nesme et al. [17] to model the small linear deformations that are necessary for sound rendering. In this approach, the object is embedded in a regular grid where each cell is a finite element, contrary to traditional FEM models where the elements try to match the object geometry as finely as possible. Tuning the grid resolution allows us to easily trade off accuracy for speed. The object is embedded in the cells using barycentric coordinates. Though the geometry of the mesh is quite different from the object geometry, the mechanical properties (mass and stiffness) of the cells match as closely as possible the spatial distribution and the parameters of material. The technique can be summarized as follows. An automatic high-resolution voxelization of the geometric object is first built. The voxelization initially concerns the surface of the geometric model, while the interior is automatically filled when the geometry represents a solid object. The voxels are then recursively merged ( 8 to 1 ) up to the desired coarser mechanical resolution. The merged voxels are used as hexahedral (boxes with the same shape ratio as the fine voxels) finite elements embedding the detailed geometric shape. The voxels are usually cubes but they may have different sizes in the three directions. At each step of the coarsification, the stiffness and mass matrices of a coarse element are computed based on the eight child element matrices. Mass and stiffness are thus deduced from a fine grid to a coarser one, where the finest depth is considered close enough to the surface, and the procedure can be described as a two-level structure, that is, from fine to coarse grid. The stiffness and mass matrices are computed bottom-up using the following equation:

$$
\mathbf{K}_{\text {parent }}=\sum_{i=0}^{7} \mathbf{L}_{i}^{T} \mathbf{K}_{i} \mathbf{L}_{i}
$$

where $\mathbf{K}$ is the matrix of the parent node, the $\mathbf{K}_{i}$ are the matrices in the child nodes, and the $\mathbf{L}_{i}$ are the interpolation matrices of the child cell vertices within the parent cell. Since empty children have null $\mathbf{K}_{i}$ matrices, the fill rate is automatically taken into account, as well as the spatial distribution of the material through the $\mathbf{L}_{i}$ matrices. As a result, full cells are heavier and stiffer than partially empty cells, and the matrices not only encode the fill rate but also the distribution of the material within each cell. With this method, we can handle objects with geometries that simultaneously include volumetric and surface parts; thin or flat features will occupy voxels and will thus result in the creation of mechanical elements that robustly approximate their mechanical behavior (see Section 5.1).

3.2. Modal Analysis. The method for FEM model [17] is adapted from realtime deformation to modal analysis. In particular, the modal parameters are extracted in a preprocessing step by solving the equation of motion for small linear deformations. We first compute the global mass and global stiffness matrices for the object by assembling the element matrices. In the case of three-dimensional objects, global matrices will have a dimension of $3 m \times 3 m$ where $m$ is the number of nodes in the finite element mesh. Each entry in each of the $24 \times 24$ element matrices for a cell is accumulated into the corresponding entry of the global matrix. Because each node in the hexahedral mesh shares an element with only a small number of the other nodes, the global matrices will be sparse. If we assume the displacements are small, the discretized system is described on a mechanical level by the Newton second law

$$
M \ddot{d}+C \dot{d}+K d=f,
$$

where $\mathbf{d}$ is the vector of node displacements, and a derivative with respect to time is indicated by an overdot. $\mathbf{M}, \mathbf{C}$, and $\mathbf{K}$ are, respectively, the system's mass, damping and 
(1) Compute mass and stiffness at desired mechanical level

(2) Assemble the mass and the stiffness matrices

(3) Modal analysis: solve the eigenproblem

(4) Store eigenvalues and eigenvectors for sound synthesis

Algorithm 1: Algorithm for modal parameters extraction.

stiffness matrices, and $\mathbf{f}$ represents external forces, such as impact forces that will produce audible vibrations. Assuming Rayleigh damping, that is, $\mathbf{C}=\alpha_{1} \mathbf{K}+\alpha_{2} \mathbf{M}$ with some $\alpha_{1}$ and $\alpha_{2}$, we can solve the eigenproblem of the decoupled system leading to the $n$ eigenvalues and the $n \times m$ matrix of eigenvectors, with $n$ the number of degrees of freedom and $m$ the number of nodes in the mesh. The sparseness of $\mathbf{M}$ and $\mathbf{K}$ matrices allows the use of sparse matrix algorithms for the eigen decomposition. We refer the reader to Appendices A and $\mathrm{B}$ for more details on the calculation.

Let $\lambda_{i}$ be the $i$ th eigenvalue and $\phi_{i}$ its corresponding eigenvector. The eigenvector, also known as the mode shape, is the deformed shape of the structure as it vibrates in the $i$ th mode. The natural frequencies and mode shapes of a structure are used to characterize its dynamic response to loads in the linear regime. The deformation of the structure is then calculated from a combination of the mode shapes of the structure using the modal superposition technique. The vector of displacements of the model, $\mathbf{u}$, is defined as:

$$
\mathbf{u}=\sum \beta_{i} \phi_{i}
$$

where $\beta_{i}$ is the scale factor for mode $\phi_{i}$. The eigenvalue for each mode is determined by the ratio of the mode's elastic stiffness to the mode's mass. For each eigen decomposition, there will be six zero eigenvalues that correspond to the six rigid-body modes, that is, modes that do not generate any elastic forces.

Our preprocessing step that performs modal analysis can be summarized as in Algorithm 1.

Our model approximates the motion of the embedded mesh vertices. That is, the visual model with detailed geometry does not match the mechanical model on which the modal analysis is performed. The motion of the embedding uses a trilinear interpolation of the mechanical degrees of freedom, so we can nevertheless compute the motion of any point on the surface given the mode shapes.

3.3. Sound Generation. In essence, efficiency of modal analysis relies on neglecting the spatial dynamics and modelling the actual physical system by a corresponding generalized mass-spring system which has the same spectral response. The activation of this model depends on where the object is hit. If we hit the object at a vibration node of a mode, then that mode will not vibrate, but others will. This is what we refer to as the sound map, which could also be called a sound excitation map as it indicates how the different modes are excited when the object is struck at different locations.

From the eigenvalues and the matrix of eigenvectors, we are able to deduce the modal parameters for sound synthesis. Let $\lambda_{i}$ be the $i$ th eigenvalue and $\omega_{i}$ its square root. The absolute value of the imaginary part of $\omega_{i}$ gives the natural frequency (in radians/second) of the $i$ th mode of the structure, whereas the real part of $\omega_{i}$ gives the mode's decay rate. The mode's gain is deduced from the eigenvectors matrix and depends on the excitation location. We refer the reader to the Appendices A and B for more details on modal superposition.

The sound resulting from an impact on a specific location $j$ on the surface is calculated as a sum of $n$ damped oscillators:

$$
s_{j}(t)=\sum_{i=1}^{n} a_{i j} \sin \left(2 \pi f_{i} t\right) e^{-d_{i} t},
$$

where $f_{i}, d_{i}$, and $a_{i j}$ are, respectively, the frequency, the decay rate and the gain of the mode $i$ at point $j$ in the sound map. An object characterized with $m$ mesh nodes and $n$ degreesof freedom is described with the vectors of frequencies and decay rates of dimension $n$, and the matrix of gains of dimension $n \times m$.

3.4. Implementation. Our deformation model implementation uses the SOFA Framework ( http://www.sofa-framework .org/) for small elastic deformations. SOFA is an open-source $\mathrm{C}++$ library for physical simulation and can be used as an external library in another program, or using one of the associated GUI applications. This choice was motivated by the ease with which it could be extended for our purpose.

Regarding sound generation, we synthesize the sounds via a reson filter (see, for example, Van den Doel et al. [6]). This choice is made based on the effectiveness for realtime audio processing. Sound radiation amplitudes of each mode is also estimated with a far-field radiation model ([15, Equation (15)]). As the motions of objects are computed with modal analysis, surfaces can be easily analyzed to determine how the motions induce acoustic pressure waves in the surrounding medium. However, we decide to focus our study on effective modal synthesis. Finally, our approach does not consider contact-position-dependent damping or changes in boundary constraints, as might happen during moments of excitation. Instead we use a uniform damping value for the sounding object.

\section{Validation of the Model}

4.1. A Metal Cube. In order to globally validate our method for modal analysis, we study the sound emitted when impacting a cube in metal. Due to its symmetry, the cube should sound the same when struck at any of the eight corners, with an excitation force whose direction is the same to the face (see Appendices A and B for more details on the force amplitude vector). We use a force normal to the face cube in order to guarantee the maximum energy in all excited modes. The sound emitted should also be similar when hitting with perpendicular forces that are both normal to one pair of the cube faces.

We suppose the cube is made of steel with Young's modulus $21 \times 10^{10} \mathrm{~Pa}$, Poisson ratio 0.33 , and density 
$7850 \mathrm{~kg} / \mathrm{m}^{3}$. The Raleigh coefficients for stiffness and mass are set to $1 \times 10^{-7}$ and 0 , respectively. The use of a constant damping ratio is a simplification that still produces good results. The cube model has edges which are 1 meter long. A Dirac is chosen for the excitation force. In this case, no radiation properties are considered.

In this example, a $3 \times 3 \times 3$ grid of hexahedral finite elements is used, leading to 192 modes. However, to adapt the stiffness of a cell according to its content, the mesh is refined more precisely than desired for the animation. The information is propagated from fine cells to coarser cells. For this example, the elements of the $3 \times 3 \times 3$ cells coarse grid resolution approximates mechanical properties propagated from a fine grid of $6 \times 6 \times 6$ cells and 216 elements (see Section 3.1 for more details on the two-level structure).

We observe in Figure 1 that the resulting sounds when impacting on different corners of the cube are identical. Also, this is true when exciting with perpendicular forces that are normal to cube faces. This shows that our model respects the symmetry of objects, as expected.

4.2. Position-Dependent Sound Rendering. To properly render impact sounds of an object, the method must preserve the sound variety when hitting the surface at different locations. We consider a metal bowl, modeled by a triangle mesh with 274 vertices, shown in Figure 2.

The material of the bowl is aluminium, with the parameters $69 \times 10^{9} \mathrm{~Pa}$ for Young's modulus, 0.33 for Poisson ratio, and $2700 \mathrm{~kg} / \mathrm{m}^{3}$ for the density. The Rayleigh damping parameters for stiffness and mass are set to $3 \times 10^{-6}$ and 0.01 , respectively. The bowl has a width of 1 meter. No radiation properties are considered; our study focuses specifically on modal synthesis.

We compare our approach to modal analysis performed first using tetrahedralization with Tetgen (http://tetgen .berlios.de/) with 822 modes. Our method uses hexahedral finite elements and is applied with a grid of $6 \times 6 \times 6$ cells, leading to 891 modes. For this example, the elements of the $6 \times 6 \times 6$ cells coarse grid resolution approximates mechanical properties propagated from a fine grid of $12 \times 12 \times 12$ cells.

We first compare the extracted modes from both methods. We observe that the ratio between frequencies and decays is the same for both methods. We then compare the synthesized sounds from both methods. We take 3 different locations, that is, top, side and bottom, on the surface of the object where the object is impacted, see Figure 2. The excitation force is modeled as a Dirac, such as a regular impact. The frequency content of the sound resulting from impact at the 3 locations on the surface is shown in Figure 3.

Each power spectrum is normalized with the maximum amplitude in order to factor out the magnitude of the impact. The eigenvalues that correspond to vibration modes will be nonzero, but for each free body in the system there will be six zero eigenvalues for the body's six rigid-body freedoms. Only the modes with nonzero eigenvalue are kept. Thus, 816 modes are finally used for sound rendering with the tetrahedralization method and 885 with our hexahedral FEM method.

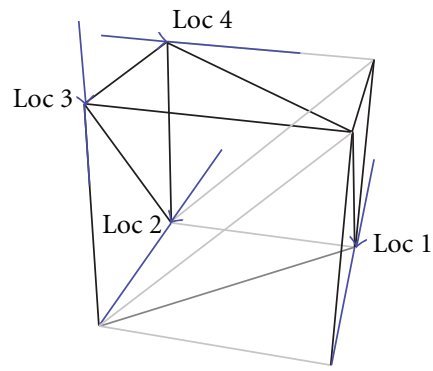

(a)
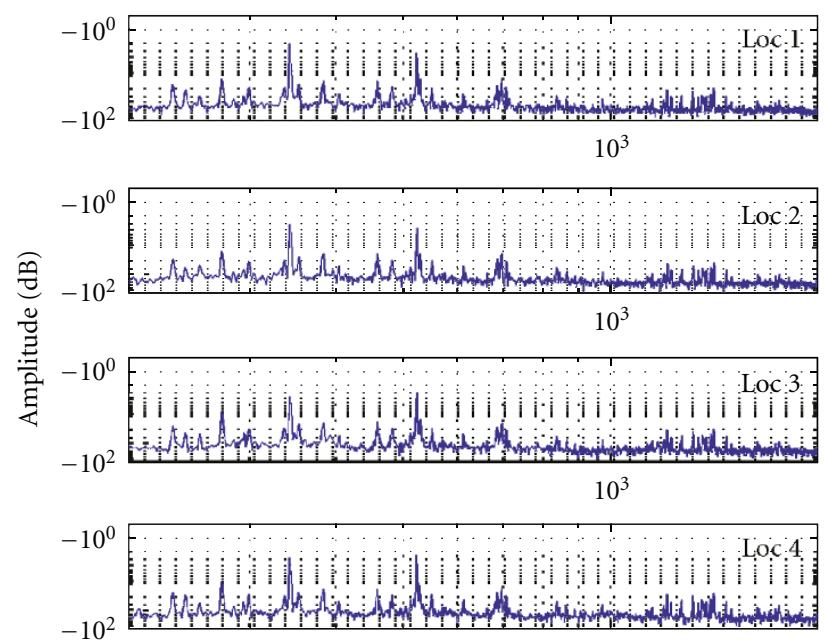

$10^{3}$

Frequency $(\mathrm{Hz})$

(b)

FIGURE 1: A sounding metal cube: sound synthesis is performed for excitation on 4 different corners and forces normal to one pair of cube faces (a); the power spectrum of the emitted sounds is given (b).

We provide with the sounds synthesized with the tetrahedral FEM and the hexadedral FEM approaches (see additional material (http://www-sop.inria.fr/members/ Cecile.Picard/Material/AdditionalMaterialEurasip.zip)).

Figure 3 highlights the similarities in the main part of the frequency content. The difference when impacting at the bottom (location 3 ) of the object is due to the difference in distribution of modes and we believe this is due to the size of the finite elements used in our method. However, we notice in listening to the synthesized sounds that those generated by our method are comparable to those created with the standard tetrahedralization.

\section{Robustness and Multiscale Results}

The number of finite elements determine the dimension of the system to solve. To avoid this expense, we provide a method that greatly simplifies the modal parameter extraction even for nonmanifold geometries. An important subclass of nonmanifold models are objects that include both volumetric and surface parts. Our technique consists of using multiresolution hexahedral embeddings. 


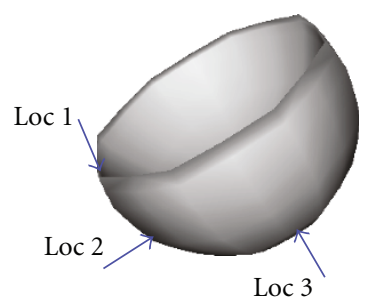

FIGURE 2: A sounding metal bowl: sound synthesis is performed for excitation on 3 specific locations on the surface.

5.1. Robustness. Most approaches for tetrahedral mesh generation have limitations. In particular, an important requirement imposed by the application of deformable FEM is that tetrahedra must have appropriate shapes, for instance, not too flat or sharp. By far the most popular of the tetrahedral meshing techniques are those utilizing the Delaunay criterion [18]. When the Delaunay criterion is not satisfied, modal analysis using standard tetrahedralization is impossible. In comparison with tetrahedralization methods, our technique can handle complex geometries and adequately performs modal analysis. Figures 4 and 5 give an example of sound modelling on a problematic geometry for tetrahedralization because of the presence of very thin parts, specifically the blades that protrude from either side.

We suppose the object is made of aluminum (see Section 4.2 for the material parameters). The object has a height of 1 meter. We apply a coarse grid of $7 \times 7 \times 7$ cells for modal analysis. The coarse level encloses the mechanical properties of a fine grid of $14 \times 14 \times 14$ cells (see Section 3.1 for more details on the two-level structure). In this example, sound radiation amplitudes of each mode are also estimated with a far-field radiation model [15, Equation (15)]. Figure 5 shows the power spectrum of the sounds resulting from impacts, modeled as a Dirac, on 6 different locations. Each power spectrum is normalized with the maximum amplitude of the spectrum in order to factor out the magnitude of the impact.

We provide with the sounds resulting when hitting on the 6 different locations (see additional material, link referred in Section 4.2). Figure 5 shows the variation of impact sounds at different surface locations due to the sound map since the different modes have varying amplitude depending on the location of excitation. The frequency content is related to the distribution of mass and stiffness along the surface and more precisely to the ratio between stiffness and mass. The similarities in the resulting sounds when hitting on location 1 and location 3 are due to the similarities of the local geometry. However, the stiffness at location 3 is smaller, allowing more resonance when being struck which explains the predominant peak in the corresponding power spectrum. When hitting the body of the object at location 2, the stiffness is locally smaller in comparison to locations 1 and 3, leading to a larger amount of low-frequency content. Also, it is interesting to examine the quality of the sound rendered when hitting the wings (locations 4,5 , and 6 ). Because wings are thin and light in comparison to the rest of the object, the higher frequencies are more pronounced. Finally, impacts on locations 2 and 4 gives comparable sounds since the impact locations are close on the body of the object.

5.2. A Multi-scale Approach. To study the influence of the number of hexahedral finite elements on sound rendering, we model a sounding object with different resolutions of hexahedral finite elements. We have created a squirrel model with 999 vertices which we use as our test sounding object. The squirrel model has a height of 1 meter. Its material is pine wood, which has parameters $12 \times 10^{9} \mathrm{~Pa}$ for Young's modulus, 0.3 for Poisson ratio, and $750 \mathrm{~kg} / \mathrm{m}^{3}$ for the volumetric mass. Rayleigh damping parameters for stiffness and mass are set to $8 \times 10^{-6}$ and 50, respectively.

Sound synthesis is performed for 3 different locations of excitation, see Figure 6 (top left). The coarse grid resolution for finite elements is set to $2 \times 2 \times 2,3 \times 3 \times 3,5 \times 5 \times 5$, and $7 \times 7 \times 7$. In this example, each grid uses mass and stiffness computed as described in Section 3.1 from a resolution 4 times finer; that is, the model with resolution $2 \times 2 \times 2$ has properties computed with a grid of $8 \times 8 \times 8$.

We provide with the sounds synthesized with the different grid resolutions for finite elements and for the 3 different locations of excitation (see additional material, link referred in Section 4.2). Results show that the frequency content of sounds depend on the location of excitation and on the resolution of the hexahedral finite elements. The higher resolution models have a wider range of frequencies because of the supplementary degrees of freedom. We also observe a frequency shift as the FEM resolution increases. Note that a $2 \times 2 \times 2$ grid represents an extremely coarse embedding, and consequently it is not surprising that the frequency content is different at higher resolution. Nevertheless, there are still some strong similarities at the dominant frequencies. Above all, a desirable feature is the convergence of frequency content as the resolution of the model increases. While additional psychoacoustic experiments with objective spectral distortion measures would be necessary to validate this result, when listening to the results, the sound quality for this model at a grid of $5 \times 5 \times 5$ may produce a convincing sound rendering for the human ear. Figure 6 suggests that higher resolutions are necessary before convergence can be clearly observed in the frequency content. Finally, we note that the grid resolution required for acceptable precision in the sound rendering depends on the geometry of the simulated object.

5.3. Discussion. The sound map is influenced by the resolution of the hexahedral finite elements. This is related to the way stiffnesses and masses of different elements are altered based on their contents. As a consequence, a $2 \times 2 \times 2$ hexahedral FEM resolution would show much less expressive variation than higher FEM resolution (we refer the reader to the records provided in the additional material, link referred in Section 4.2). One approach to improving this would be to use better approximations of the mass and stiffness of coarse elements [19].

Modelling numerous complex sounding objects can rapidly become prohibitively expensive for realtime 

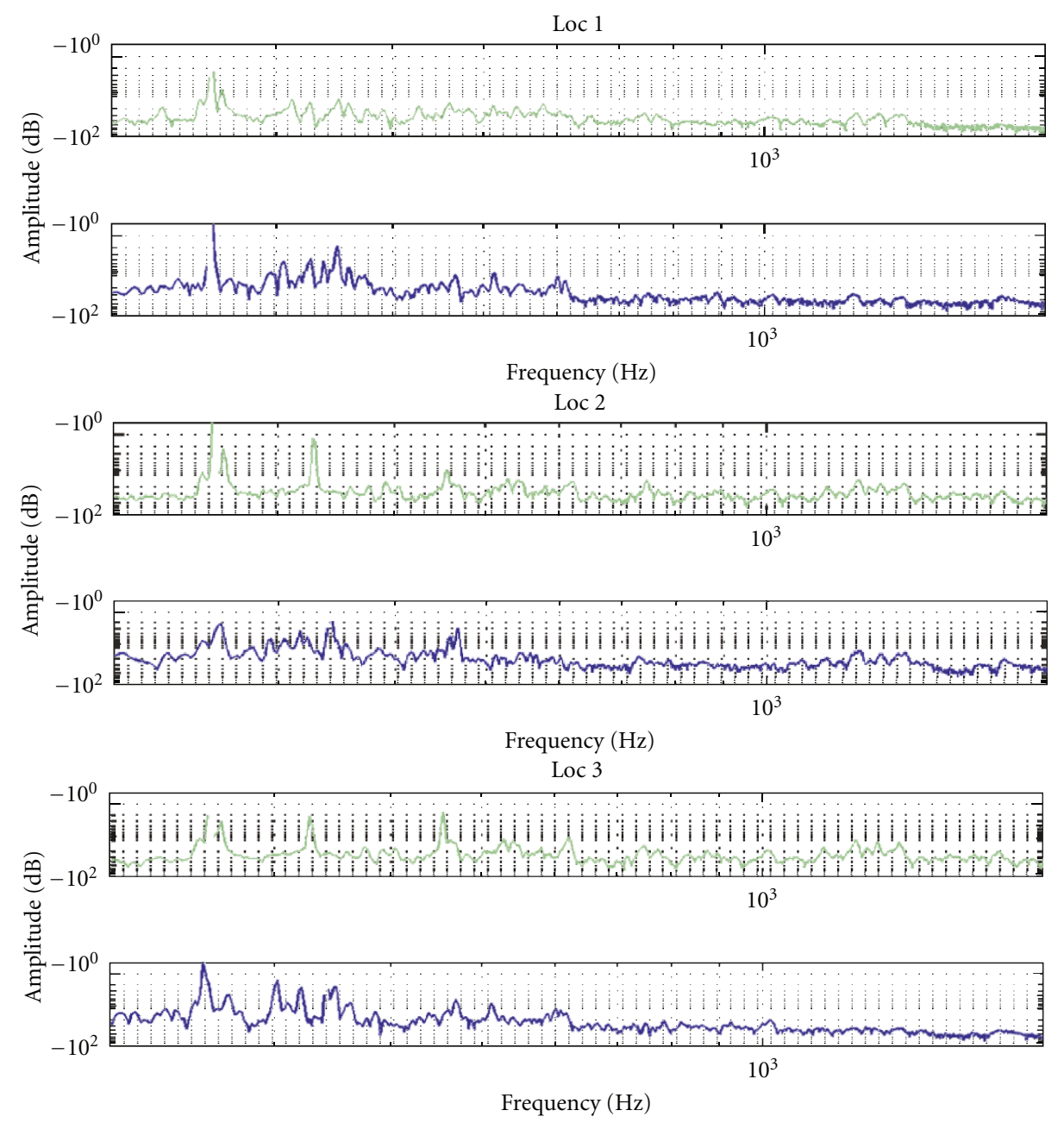

FIGURE 3: Sound synthesis with a modal approach using classical tetrahedralization with 822 modes (green) and our method with a $6 \times 6 \times 6$ hexahedral FEM resolution, leading to 891 modes (blue): power spectrum of the sounds emitted when impacting at the 3 different locations shown in Figure 2.
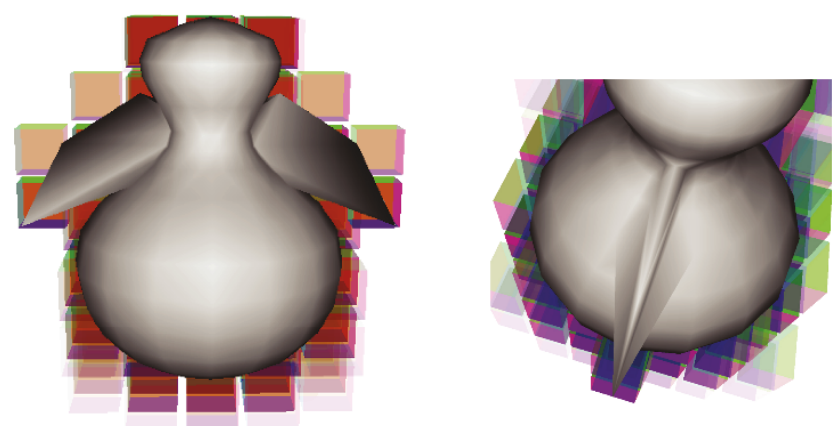

Figure 4: An example of a complex geometry that can be handled with our method. The thin blade causes problems with traditional tetrahedralization methods.

rendering due to the large set of modal data that has to be handled. Nevertheless, based on the quality of the resulting sounds obtained with our method, and given that increased resolution for the finite elements implies higher memory and computational requirements for modal data, the FEM resolution can be adapted to the number of sounding objects in the virtual scene.

Table 1 gives the computation times and the memory usage of the modal data, that is, frequencies, decay rates and gains, when computing the modal analysis with different FEM resolution on the squirrel model. In this example, the finer grid resolution is two levels up to the one of coarse grid, that is, a coarse grid of $2 \times 2 \times 2$ cells has a fine level of $8 \times 8 \times 8$ cells with 337 degrees of freedom ( 3954 for $5 \times 5 \times 5$ ). These are computation times of an unoptimized implementation on a $2.26 \mathrm{GHz}$ Intel Core Duo. We highlight the $5 \times 5 \times 5$ cells resolution since the results indicate that this resolution may be sufficient to properly render the sound quality of the object (see Section 5.2). These results could be improved by reformulating the computations in order to be supported by graphics processing units (GPU).

Despite the fact that audio is considered a very important aspect in virtual environments, it is still considered to be of lower importance than graphics. We believe that physically modeled audio brings a significant added value in terms 

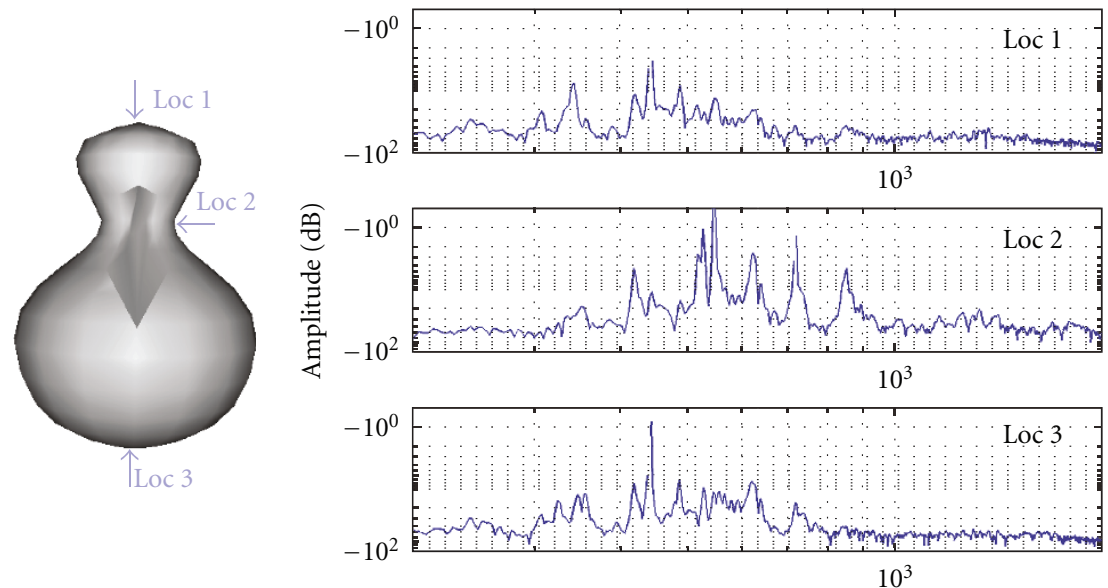

$10^{3}$
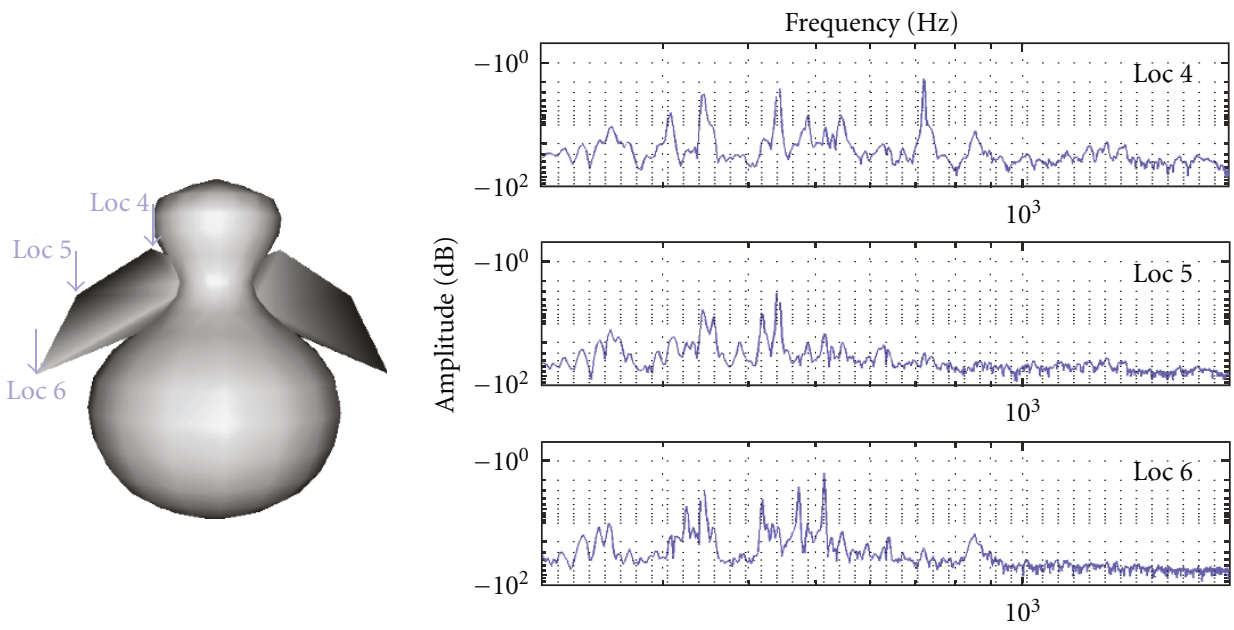

Frequency $(\mathrm{Hz})$

Figure 5: The power spectrum of the sounds resulting from impacts at the 3 different locations on the body of the object (top) and on the 3 different locations on the wing (bottom). Note that the audible response is different based on where the object is hit.

TABle 1: Computation times in seconds and memory usage in megabytes for different grid resolutions. Computation times are given for the different steps of the calculation: discretization and computation of mass and stiffness matrices (T1), eigenvalues extraction (T2), and gains computation (T3).

\begin{tabular}{lcccccc}
\hline $\begin{array}{l}\text { Grid Res. } \\
\text { (cells) }\end{array}$ & \# Modes & $\begin{array}{c}\text { T1 } \\
(\mathrm{s})\end{array}$ & $\begin{array}{c}\text { T2 } \\
(\mathrm{s})\end{array}$ & $\begin{array}{c}\text { T3 } \\
(\mathrm{s})\end{array}$ & $\begin{array}{c}\text { Total } \\
(\mathrm{s})\end{array}$ & $\begin{array}{c}\text { MEM } \\
(\mathrm{MB})\end{array}$ \\
\hline $7 \times 7 \times 7$ & 1191 & 1.81 & 16.06 & 3.99 & 21.89 & 9.3 \\
$\mathbf{6} \times \mathbf{6} \times \mathbf{6}$ & 846 & 0.89 & 5.78 & 2.39 & 9.06 & 6.8 \\
$\mathbf{5} \times \mathbf{5} \times \mathbf{5}$ & $\mathbf{5 7 9}$ & $\mathbf{0 . 4 3}$ & $\mathbf{2 . 0 7}$ & $\mathbf{0 . 9 7}$ & $\mathbf{3 . 4 7}$ & $\mathbf{4 . 7}$ \\
$4 \times 4 \times 4$ & 363 & 0.24 & 0.61 & 0.59 & 2.88 & 2.9 \\
$3 \times 3 \times 3$ & 192 & 0.05 & 0.14 & 0.16 & 0.35 & 1.6 \\
$2 \times 2 \times 2$ & 81 & 0.01 & 0.03 & 0.01 & 0.05 & 0.69 \\
\hline
\end{tabular}

of realism and the increased sense of immersion. Our method is built on a physically based animation engine, the SOFA Framework. As a consequence, problems of coherence between physics simulation and audio are avoided by using exactly the same model for simulation and sound modelling.
The sound can be processed in realtime knowing the modal parameters of the sounding object.

\section{Experimenting with the Modal Sounds in Realtime}

To apply excitation signals in realtime to the simulated sounding objects, we implemented an object, or data processing block, for Pure Data and Max/MSP, two similar visual programming modular environments for dataflow processing. We used the flext library (http://puredata.info/ Members/thomas/flext/) (API for object development common to both environments), and the $\mathrm{C} / \mathrm{C}++$ code for modal synthesis of bell sounds from van den Doel and Pai [20]. The object in use on a Pure Data patch is illustrated in Figure 7). We provide the user two different ways for the user to interact with the model. The user can either choose a specific mesh vertex number of the geometry model (represented in red in the figure), or can choose a specific location (in green) where the nearest vertex is deduced by interpolation.

One advantage of the method is to give the possibility to control the parameters of the sounding model in order 

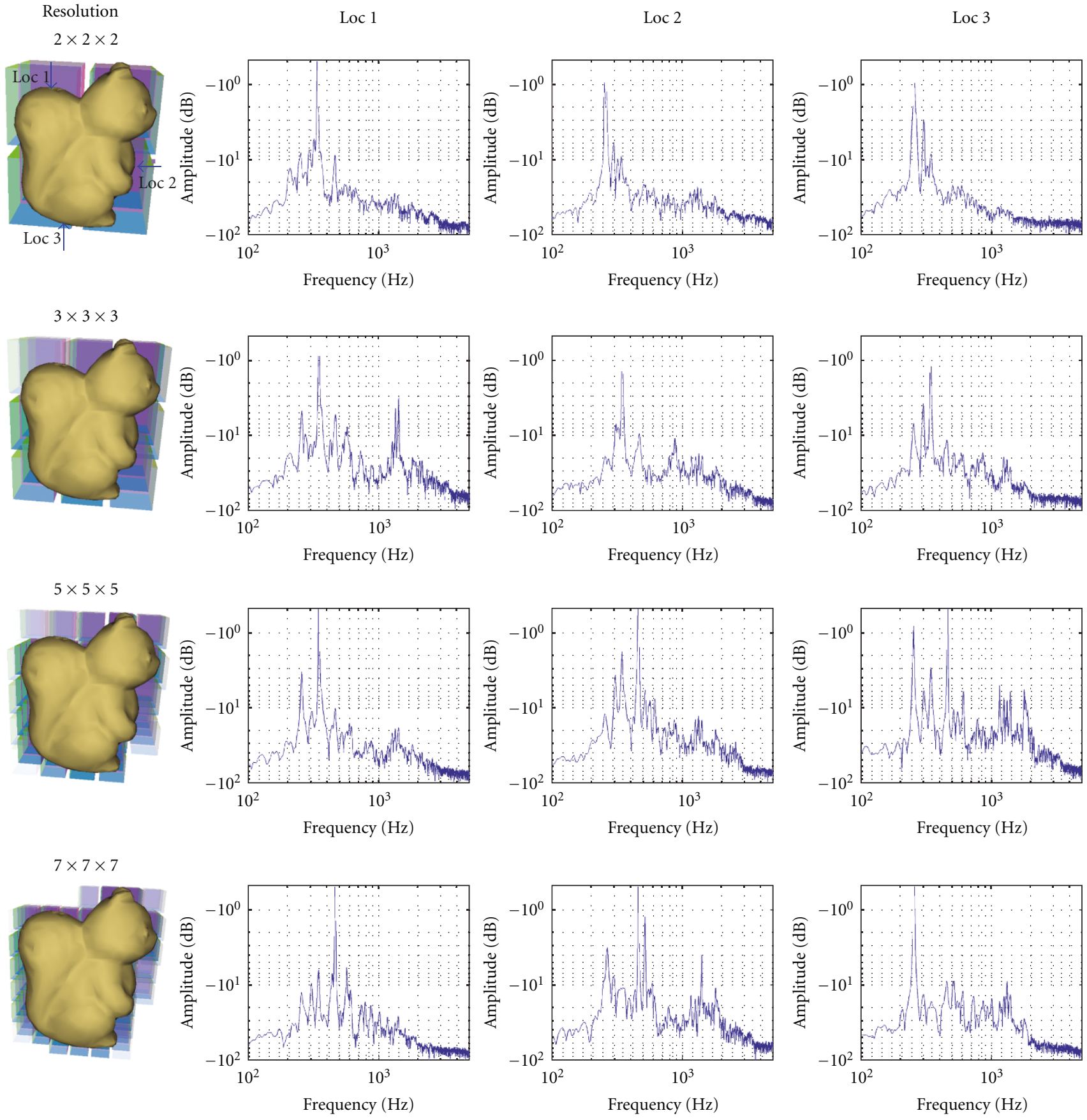

FIGURE 6: A squirrel in pine wood is sounding when struck at 3 different locations (from left to right). Frequency content of the resulting sounds with 4 different resolutions for the hexahedral finite elements: (from top to bottom), $2 \times 2 \times 2,3 \times 3 \times 3,5 \times 5 \times 5,7 \times 7 \times 7$ cells.

to tune the resulting sounds for the desired effect. For instance, the size of the geometry can be modified as different dimensions could be preferred for rendering sounds in a particular scenario. The mesh geometry is loaded in Alias $\backslash$ Wavefront ${ }^{*}$.obj format, and we use Blender to apply geometrical transformations in order to test how it affects the rendering of the resulting sounds.

As our sound model consists of an excitation and a resonator, interesting sounds can be easily obtained by convolving modal sounds with user-defined excitations. The excitation which supplies the energy to the sound system contributes to a great extent to the fine details of the resulting sounds. Excitation signals may be produced by various ways: loading recorded sound samples, using realtime signals coming from live soundcard inputs, connecting the output of other audio applications with Pure Data through a sound server.

This interface can be viewed as a preliminary prototyping tool for sound design. Indeed, by experimenting sounds with predefined objects and interactions types, the parameters of 


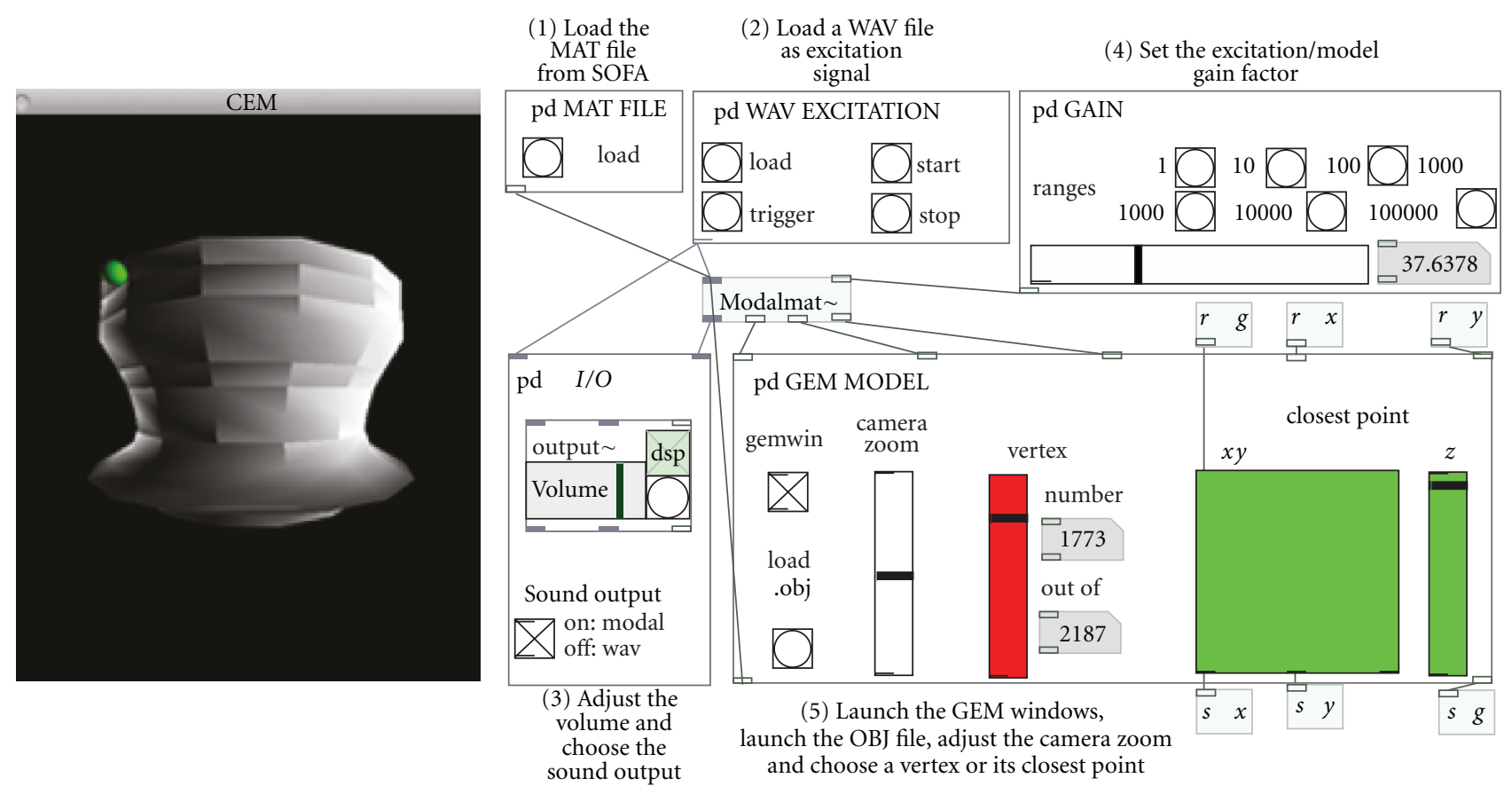

FIGURE 7: Interface for sound design. After having loaded the modal data and the corresponding mesh geometry, the user can experiment the modal sounds when exciting the object surface at different locations. Excitation signals may be loaded as recorded sound samples or realtime tracked from live soundcard inputs.

sounding objects can easily chosen in order to convey specific sensations in games. Our approach offers a great extent of control regarding the possibilities of sound modification, towards a wide audience since its implementation is crossplatform and open source. In [21], Bruyns proposed an AudioUnit plugin, that is unfortunately no longer available, for modal synthesis of arbitrarily shaped objects, where materials could be changed based on interpolation between precalculated variations on the model. Lately, Menzies has introduced VFoley in [22], an opensource environment for modal synthesis of 3D scenes, with consequent options on parameterization (particularly with many collision and surface models), but tied to physically plausible sounds as opposed to physically-inspired sounds. This is shown in the movie provided as additional material (see link referred in Section 4.2).

\section{Conclusion}

We propose a new approach to modal analysis using automatic voxelization of a surface model and computation of the finite elements parameters, based on the distribution of material in each cell. Our goal is to perform sound rendering in the context of an animated realtime virtual environment, which has specific requirements, such as realtime processing and efficient memory usage.

For simple cases, our method gives results similar to traditional modal analysis with tetrahedralization. For more complex cases, our approach provides convincing results. In particular, sound variety along the object surface, the sound map, is well preserved. Our technique can handle complex nonmanifold geometries that include both volumetric and surface parts, which cannot be handled by previous techniques. We are thus able to compute the audio response of numerous and diverse sounding objects, such as those used in games, training simulations, and other interactive virtual environments. Our solution allows a multiscale solution because the number of hexahedral finite elements only loosely depends on the geometry of the sounding object. Finally, since our method is built on a physics animation engine, the SOFA Framework, problems of coherence between simulation and audio can be easily addressed, which is of great interest in the context of interactive environment.

In addition, due to the fast computation time, we are hopeful that realtime modal analysis will soon be possible on the fly, with sound results that are approximate but still realistic for virtual environments. For this purpose, psychoacoustic experiments should be conducted to determine the resolution level for acceptable quality of the sound rendering.

\section{Appendices}

These appendices give the mathematical background behind modal superposition for discrete systems with proportional damping. To apply modal superposition, we assume the steady state situation, that is, the sustained part of the impulse response of an object being struck. Indeed, the early part, which is of very short duration, contains many frequencies and is consequently not well described by a discrete set of frequencies. Modal superposition uses the 
Finite Element Method (FEM) and determine the impulse response of vibrating objects by means of a superposition of eigenmodes.

\section{A. Derivation of the Equations}

We first consider the undamped system; its equation of motion is expressed by

$$
\mathbf{M x}+\mathbf{K x}=\mathbf{f},
$$

where $\mathbf{M}$ and $\mathbf{K}$ are, respectively, the mass and stiffness matrices of the discrete system. The mass matrix is typically a diagonal matrix, its main diagonal being populated with elements whose value is the mass assumed in each degree of freedom (DOF). The stiffness matrix is symmetric (often a sparse matrix, that is, only a band of elements around the main diagonal is populated and the other elements are zero). In finite elements, these matrices are assembled based on the element geometry and properties.

Since the study is in the frequency domain, the displacement vector $\mathbf{x}$ and the force vector $\mathbf{f}$ are based on harmonic components, that is, $\mathbf{x}=\mathbf{X} e^{j \omega t}, \dot{\mathbf{x}}=j \omega \mathbf{X} e^{j \omega t}, \ddot{\mathbf{x}}=-\omega^{2} \mathbf{X} e^{j \omega t}$ and $\mathbf{f}=\mathbf{F} e^{j \omega t}$. $\mathbf{X}$ and $\mathbf{F}$ are two amplitude vectors and contain one element for each degree of freedom (DOF). The elements of $\mathbf{X}$ are the displacement amplitudes of the respective DOF as a function of $\omega$ and the elements of $\mathbf{F}$ are the amplitudes of the force, again depending on $\omega$, acting at location and in direction of the corresponding DOF. Since the harmonic part is available on both sides, we can ignore it and the equation of motion can be rewritten

$$
\mathbf{X}=\left(\mathbf{K}-\omega^{2} \mathbf{M}\right)^{-1} \mathbf{F},
$$

where $\mathbf{X}$ and $\mathbf{F}$ means in practice $\mathbf{X}(\omega)$ and $\mathbf{F}(\omega)$, but are shorten for simplification, and $\omega$ is a diagonal matrix. Equation (A.2) is the direct frequency response analysis. The term $\mathbf{K}-\omega^{2} \mathbf{M}$ needs to be calculated for each frequency. To calculate the response to any excitation force $\mathbf{F}(\omega)$, we need to solve the eigenvalue problem:

$$
\left(\mathbf{K}-\omega^{2} \mathbf{M}\right) \mathbf{X}=0,
$$

or

$$
\left(\mathbf{M}^{-1} \mathbf{K}\right) \mathbf{X}=\omega^{2} \mathbf{X}=\lambda \mathbf{X} .
$$

This equation says that each sounding object has a structurerelated set of eigenvalues $\lambda$, which are simply connected to the system's frequencies. To extract the eigenvalues, the following condition has to be fulfilled

$$
\operatorname{det}\left(\mathbf{K}-\omega^{2} \mathbf{M}\right)=0 .
$$

Solving (A.5) implies finding the roots of a polynomial, which correspond to the eigenvalues $\lambda$. The latter can then be replaced in (A.3):

$$
(\mathbf{K}-\lambda \mathbf{M}) \Psi=0,
$$

$\Psi$ is the matrix of eigenvectors, or eigenfunctions, where the column $\mathbf{r}$ is the vector related to the eigenvalue $\omega_{r}^{2}$.
The eigenvectors define the mode shapes linked to the corresponding frequency of the system.

If the frequencies are unique, many eigenvectors can be extracted for a given eigenvalue and all are proportional. Thus, the information enclosed in the eigenvectors is not the absolute amplitude but a ratio between the amplitudes in the degrees of freedom. For this reason, the eigenvectors are often normalized according to a reference. Due to the orthogonal property of the eigenvectors, $\Psi^{T} \Psi=\mathbf{I}$. Consequently, $\Psi^{T} \mathbf{M} \Psi$ and $\Psi^{T} \mathbf{K} \Psi$ are diagonal matrices, and are, respectively, called the modal mass and the modal stiffness of the system, because the ratio between modal stiffness and modal mass gives the matrix of eigenvalues. A very suitable reference choice is to scale the eigenvectors so that the modal mass matrix becomes an identity matrix. From (A.2), we can write:

$$
\begin{gathered}
\Psi^{T}\left(\mathbf{K}-\omega^{2} \mathbf{M}\right) \Psi=\Psi^{T} \frac{\mathbf{F}(\omega)}{\mathbf{X}(\omega)} \Psi, \\
\left(\lambda-\omega^{2} \mathbf{I}\right)=\Psi^{T} \frac{\mathbf{F}(\omega)}{\mathbf{X}(\omega)} \Psi,
\end{gathered}
$$

and finally

$$
\mathbf{X}(\omega)=\Psi\left(\lambda-\omega^{2} \mathbf{I}\right)^{-1} \Psi^{T} \mathbf{F}(\omega) .
$$

Equation (A.8) simply expresses that the response $\mathbf{X}(\omega)$ can be calculated by surimposing a set of eigenmodes weighted by the excitation frequency, multiplied with an excitation load vector $\mathbf{F}(\omega)$.

Properties of Eigenvalues and Eigenvectors. The orthogonality of modes expresses that each mode contains information which the other modes do not have, and consequently a given mode cannot be built from the others. On the other hand, solutions of geometrically symmetric systems often give pairs of multiple eigenmodes.

Boundary conditions are settled simply by prescribing the value of certain degrees of freedom resolved in the displacement vector. As an example, a structure rigidly attached to the ground will show null DOFs around the support point. Consequently, the elements in the mode shapes corresponding to these DOFs will always be zero and will not need to be solved.

\section{B. Damping}

We now consider a damped system, and in particular the proportional damping model which assumes that the damping can be expressed proportional to the stiffness and mass matrix (Raleigh damping), that is, $\mathbf{C}=\alpha_{1} \mathbf{K}+\alpha_{2} \mathbf{M}$. In consequence, the eigenvalues of the proportional damped system are complex and can be expressed according to the eigenvalues of the undamped case

$$
\lambda_{r}^{\prime}=\omega_{r}^{2}\left(1+j \eta_{r}\right)
$$

where the imaginary part contains the loss factor $\eta_{r}$.

The modal superposition is thus given by

$$
\mathbf{X}(\omega)=\Psi\left(\lambda-\omega^{2} \mathbf{I}+j \eta \lambda\right)^{-1} \Psi^{T} \mathbf{F}(\omega) .
$$


Equation (B.2) enables us to determine entire response velocity fields that cause the surrounding medium to vibrate and to generate sound.

\section{Acknowledgments}

This work was partly funded by Eden Games (http://www .eden-games.com/), an ATARI Game Studio in Lyon, France. C. Frisson is supported by numediart (http://www.numediart.org/), a long-term research program centered on digital media arts, funded by Région Wallonne, Belgium (Grant no. 716631). The authors would like to thank Nicolas Tsingos for his input on an early draft. Special thanks to Michaël Adam and Florent Falipou for their expertise in the SOFA Framework.

\section{References}

[1] J. F. O’Brien, C. Shen, and C. M. Gatchalian, "Synthesizing sounds from rigid-body simulations," in Proceedings of ASM SIGGRAPH Symposium on Computer Animation, pp. 175-181, July 2002.

[2] J.-M. Adrien, “The missing link: modal synthesis," in Representations of Musical Signals, pp. 269-298, MIT Press, Cambridge, Mass, USA, 1991.

[3] C. Cadoz, A. Luciani, and J. Florens, "Responsive input devices and sound synthesis by simulation of instrumental mechanisms: the CORDIS system," Computer Music Journal, vol. 8, no. 3, pp. 60-73, 1984.

[4] F. Iovino, R. Caussé, and R. Dudas, "Recent work around modalys and modal synthesis," in Proceedings of the International Computer Music Conference (ICMC '97), pp. 356-359, 1997.

[5] P. R. Cook, Real Sound Synthesis for Interactive Applications, A. K. Peters, 2002.

[6] K. Van den Doel, P. G. Kry, and D. K. Pai, "Foley automatic: physically-based sound effects for interactive simulation and animation," in Proceedings of the Computer Graphics Annual Conference (SIGGRAPH '01), pp. 537-544, August 2001.

[7] N. Raghuvanshi and M. C. Lin, "Interactive sound synthesis for large scale environments," in Proceedings of ACM SIGGRAPH Symposium on Interactive 3D Graphics and Games (I3d '06), pp. 101-108, March 2006.

[8] C. Bruyns-Maxwell and D. Bindel, "Modal parameter tracking for shape-changing geometric objects," in Proceedings of the 10th International Conference on Digital Audio Effects (DAFx'07), 2007.

[9] Q. Zhang, L. Ye, and Z. Pan, "Physically-based sound synthesis on GPUs," in Proceedings of the 4th International Conference on Entertainment Computing, vol. 3711 of Lecture Notes in Computer Science, pp. 328-333, 2005.

[10] N. Bonneel, G. Drettakis, N. Tsingos, I. Viaud-Delrnon, and D. James, "Fast modal sounds with scalable frequencydomain synthesis," in Proceeding of ACM International Conference on Computer Graphics and Interactive Techniques (SIGGRAPH '08), August 2008.

[11] D. Menzies, "Physical audio for virtual environments, phya in review," in Proceedings of the International Conference on Auditory Display (ICAD), G. P. Scavone, Ed., pp. 197-202, Schulich School of Music, McGill University, 2007.
[12] J. F. O’Brien, P. R. Cook, and G. Essl, "Synthesizing sounds from physically based motion," in Proceedings of the ACM SIGGRAPH Conference on Computer Graphics, pp. 529-536, Los Angeles, Calif, USA, August 2001.

[13] R. J. McAulay and T. F. Quatieri, "Speech analysis/ synthesis based on a sinusoidal representation," IEEE Transactions on Acoustics, Speech and Signal Processing, vol. 34, no. 4, pp. 744754, 1986.

[14] K.-J. Bathe, Finite Element Procedures in Engineering Analysis, Prentice-Hall, Englewood Cliffs, NJ, USA, 1982.

[15] D. L. James, J. Barbič, and D. K. Pai, "Precomputed acoustic transfer: output-sensitive, accurate sound generation for geometrically complex vibration sources," ACM Transactions on Graphics, vol. 25, no. 3, pp. 987-995, 2006.

[16] J. N. Chadwick, S. S. An, and D. L. James, "Harmonic shells: a practical nonlinear sound model for near-rigid thin shells," in Proceedings of ACM Transactions on Graphics, 2009.

[17] M. Nesme, Y. Payan, and F. Faure, "Animating shapes at arbitrary resolution with non-uniform stiffness," in Proceedings of Eurographics Workshop in Virtual Reality Interaction and Physical Simulation (VRIPHYS '06), 2006.

[18] J. R. Shewchuk, "A condition guaranteeing the existence of higher-dimensional constrained Delaunay triangulations," in Proceedings of the 14th Annual Symposium on Computational Geometry, pp. 76-85, June 1998.

[19] M. Nesme, P. G. Kry, L. Jeřábková, and F. Faure, "Preserving topology and elasticity for embedded deformable models," ACM Transactions on Graphics, vol. 28, no. 3, article no. 52, 2009.

[20] K. van den Doel and D. K. Pai, "Modal Synthesis for Vibrating Objects," in Audio Anecdotes III: Tools, Tips, and Techniques for Digital Audio, pp. 99-120, A. K. Peter, 3rd edition, 2006.

[21] C. Bruyns, "Modal synthesis for arbitrarily shaped objects," Computer Music Journal, vol. 30, no. 3, pp. 22-37, 2006.

[22] D. Menzies, "Phya and vfoley, physically motivated audio for virtual environments," in Proceedings of the 35th AES International Conference, 2009. 